\section{Criterios de médicos tratantes para indicar instalación de gastrostomía en pacientes con demencia avanzada}

\author{
MARÍA BERNARDITA PORTALES V. ${ }^{1}$
}

\section{A survey about the criteria for the indication of a gastrostomy in patients with advanced dementia}

\begin{abstract}
Background: Decision making concerning patients with advanced dementia is complicated. The indication of a gastrostomy is among these hard decisions, especially considering that there is no consensus about its real benefit. Aim: To explore the criteria used by Chilean physicians to indicate a gastrostomy in patients with advanced dementia. Material and Methods: A questionnaire about the decision making to indicate a gastrostomy was delivered to 72 physicians working in public and private hospitals. Results: The survey was answered by 43 physicians. Fifty one percent had indicated a gastrostomy to patients with advanced dementia in the last year, 79\% believed that gastrostomy reduces the risk of aspiration pneumonia, 50\% thought that gastrostomy helps in bed sore healing and $74 \%$ believed that gastrostomy improves survival. Conclusions: The majority of physicians who answered the survey think that gastrostomy will improve the health status of patients with advanced dementia.
\end{abstract}

(Rev Med Chile 2015; 143: 1405-1410)

Key words: Bioethics; Dementia; Gastrostomy.

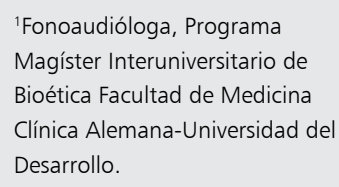

Recibido el 16 de enero de 2015 aceptado el 29 de julio de 2015

\section{Correspondencia a:}

María Bernardita Portales V.

berniportales@gmail.com

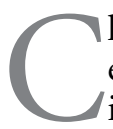
hile, así como en el resto del mundo, ha experimentado cambios poblacionales cándose una esperanza de vida 79 años $^{1} y$ el incremento de la población de adultos mayores. En este contexto, las demencias se han transformado en uno de los principales problemas de salud en este grupo poblacional. En Chile se estima que cerca de 200.000 personas presentan demencia en la actualidad y se proyecta que esta prevalencia se triplicará para el año $2050^{2}$.

La disfagia es un síndrome que comúnmente presentan los pacientes con demencia y tiene enorme impacto en la capacidad funcional, calidad de vida y salud de los pacientes que la padecen ${ }^{3}$. Los pacientes con demencia desarrollan problemas en su alimentación, declive severo en la ingesta oral, imposibilidad de comer por sí solos, resistencia/ indiferencia a la ingesta de alimentos, falla en la coordinación de la masticación y deglución del bolo alimenticio, protección de vía aérea inconsistente o aspiración al momento de deglutir ${ }^{3-5}$. Con el avance de la demencia las dificultades en la deglución convierten en problemáticos los momentos de ingesta, con el resultado de déficit en los requerimientos calóricos, pérdida de peso y malnutrición.

Muchas veces se plantea la alimentación por gastrostomía (GTT) para compensar los efectos de la disfagia en la última etapa de la demencia, pero distintos estudios sugieren que hay escasa o ninguna evidencia de que la gastrostomía mejore los marcadores nutricionales, reduzca la incidencia de neumonía, contribuya a una mejora en las úlceras por presión, o aumente la expectativa de vida en estos pacientes. A pesar de que la litera- 
tura sugiere un beneficio discutible asociado a la alimentación por GTT en pacientes en etapa avanzada de demencia, la mayoría de los médicos y otros profesionales de la salud han reportado que ellos creen que la instalación de GTT puede beneficiar a estos pacientes ${ }^{6}$.

Esta investigación, como estudio descriptivo, tiene por objetivo identificar en nuestro medio los criterios de indicación de GTT percutánea en pacientes con demencia avanzada por parte de médicos tratantes y determinar si existen diferencias en los criterios entre los médicos entrevistados según el subsistema de salud en que se desempeñen y según su especialidad.

\section{Material y Método}

Este estudio es de tipo observacional, descriptivo, de corte transversal y enfoque cuantitativo.

\section{Población y muestra}

La población estuvo compuesta por médicos neurólogos, broncopulmonares, geriatras, y aquellos que realizan turnos en la unidad de pacientes críticos (UCI e Intermedio) de una clínica privada $y$ un hospital en alianza con la anterior en Santiago de Chile. No existieron criterios de inclusión ni exclusión. La muestra es no probabilística, correspondiendo a una muestra de voluntarios, donde los médicos contestaron libremente el cuestionario. Se entregó el cuestionario a 72 médicos y se logró una muestra de 43 cuestionarios contestados (tasa de respuesta de 59,7\%).

\section{Variables e instrumento}

Se identificaron las siguientes variables: experiencia, opinión y práctica de los médicos en relación a la indicación de GTT a pacientes con demencia avanzada. Se creó un cuestionario basándose en un estudio realizado a médicos de atención primaria en Estados Unidos de Norteamérica por Shega, que trataba de comprender más acerca de las decisiones de los médicos al recomendar la colocación de GTT en pacientes con demencia avanzada ${ }^{7}$. El cuestionario fue sometido a juicio de expertos, impreso y luego entregado a los médicos. En la primera parte del instrumento se explicitaba que la participación era voluntaria. El proyecto de investigación junto con el cuestionario fue revisado y aprobado por el Comité de Ética de Investigación de la Universidad del Desarrollo-Clínica Alemana. El estudio resguardó la voluntariedad de la participación y el anonimato de las respuestas de los participantes.

\section{Análisis de resultados}

El análisis estadístico se realizó usando el programa SPSS versión 20. Se consideraron dos niveles de análisis. En primer lugar, se desarrolló un análisis univariado, calculándose frecuencias y porcentajes para las variables categóricas. Luego se llevó a cabo un análisis bivariado, comparando los datos obtenidos según dos variables de interés: la especialidad y el lugar de trabajo.

Por el tamaño de la muestra, las especialidades se agruparon en Medicina Interna y Neurología. Por su parte, se separó el sistema de trabajo en subsistema privado y público/mixto (privado y público).

Respecto a la escala de respuesta de la mayoría de las preguntas de opinión, se decidió unir las respuestas afirmativas (definitivamente sí, probablemente sí) y aquellas negativas (probablemente no y definitivamente no), generando una escala dicotómica ("sí/no"), para facilitar su análisis bivariado. En este nivel de análisis se generaron tablas de contingencia, aplicándose las pruebas de Fisher o de $\chi^{2}$ para evaluar diferencias entre los grupos, considerándose un $\alpha$ de 0,05 .

\section{Resultados}

Las características de los médicos que participaron en el estudio se resumen en la Tabla 1.

Entre los aspectos generales consultados, $76,7 \%$ de los médicos ha indicado GTT en el último año, con una tendencia a mantener el número de indicaciones en los últimos 5 años $(66,7 \%) \sin$ observarse diferencia significativa entre los subsistemas de salud ni por especialidad de los médicos. El 51,2\% indicó GTT a "pacientes con demencia avanzada" y 44,2\% señaló haber revisado literatura actual sobre GTT y demencia.

Los médicos describieron una serie de beneficios de la GTT en pacientes con demencia avanzada: $79,1 \%$ cree que disminuye la incidencia de neumonía aspirativa, $50 \%$ está de acuerdo en que la GTT mejora la curación de las úlceras por presión, 73,8\% plantea que mejora la sobrevida, 95,3\% está de acuerdo en que mejora el estado 
nutricional. Sólo 44,2\% está de acuerdo en creer que la GTT mejora el estado funcional en estos pacientes.

En las variables de opinión, se vio una diferencia significativa entre las respuestas de los médicos que trabajan en el subsistema de salud privado y mixto, observándose en el privado una mayor adherencia en afirmar que la GTT disminuye

Tabla 1. Resumen de Características de los Médicos Participantes

\begin{tabular}{|lc|}
\hline Características & $\%$ \\
\hline Género & \\
Mujeres & 44,2 \\
Hombres & 55,8 \\
\hline Lugar de trabajo & \\
Privado & 53,5 \\
Público/mixto & 46,5 \\
Especialidad & \\
Medicina Interna (23) & \\
- Geriatra (2) & 53,5 \\
- Broncopulmonar (5) & \\
- Internista con turno en UPC (16) & \\
Neurólogos (20) & \\
Años de experiencia & 46,5 \\
Menos de 10 años & \\
Más de 10 años & 41,9 \\
\hline
\end{tabular}

la incidencia de neumonía aspirativa, mejora la sobrevida y el estado funcional (Figura 1).

$\mathrm{Al}$ analizar por especialidad, sólo se observó diferencia significativa relacionada a la incidencia de neumonía aspirativa. El 100\% de los neurólogos y $60,9 \%$ de los internistas creen que la GTT disminuye su incidencia (Figura 2). El análisis de las respuestas de opinión excluyendo a los geriatras, no modificó la diferencia los resultados. Sólo existió una variación en el porcentaje que opina que la GTT disminuye la incidencia de neumonía $(66,7 \%)$, pero la diferencia por especialidad sigue siendo significativa.

Otros antecedentes que consideran los médicos al indicar GTT en pacientes con demencia avanzada son: voluntades anticipadas $(63,2 \%)$, preferencias de la familia $(94,9 \%)$, nivel cognitivo del paciente $(94,4 \%)$, pronóstico $(94,9 \%)$, funcionamiento físico $(92,3 \%)$, edad $(79,5 \%)$, costo de la GTT $(53,8 \%)$. En ninguno de estos factores hay diferencias por subsistema de salud ni por especialidad de los médicos participantes (Tabla 2).

No se observaron diferencias significativas en los ítems mencionados, pero llama la atención que los internistas consideran con mayor frecuencia las voluntades anticipadas, las preferencias de la familia, nivel cognitivo, pronóstico y funcionamiento físico del paciente. Los neurólogos consideran más la edad y el costo de la GTT. Sólo en este último

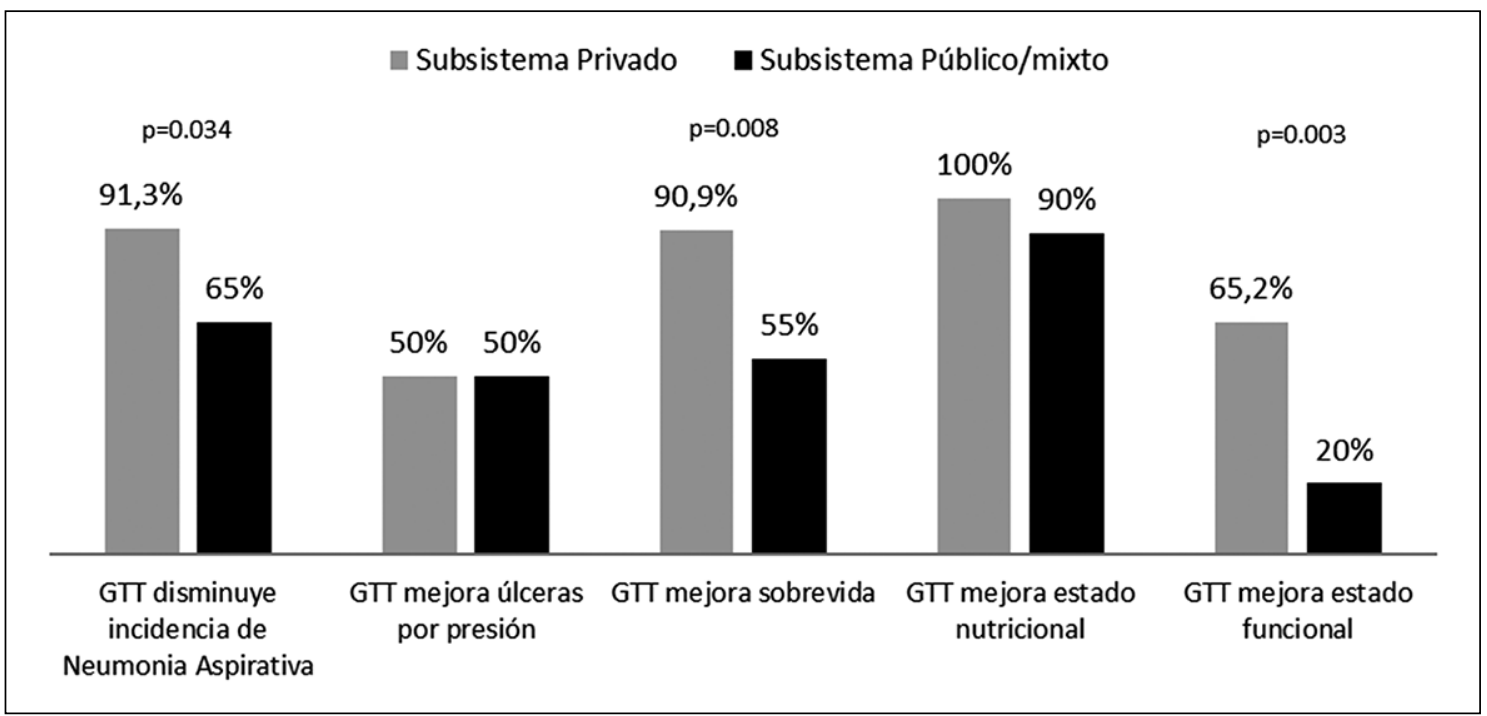

Figura 1. Resumen de opiniones sobre beneficios de gastrostomía en pacientes con demencia avanzada según subsistema de salud. 


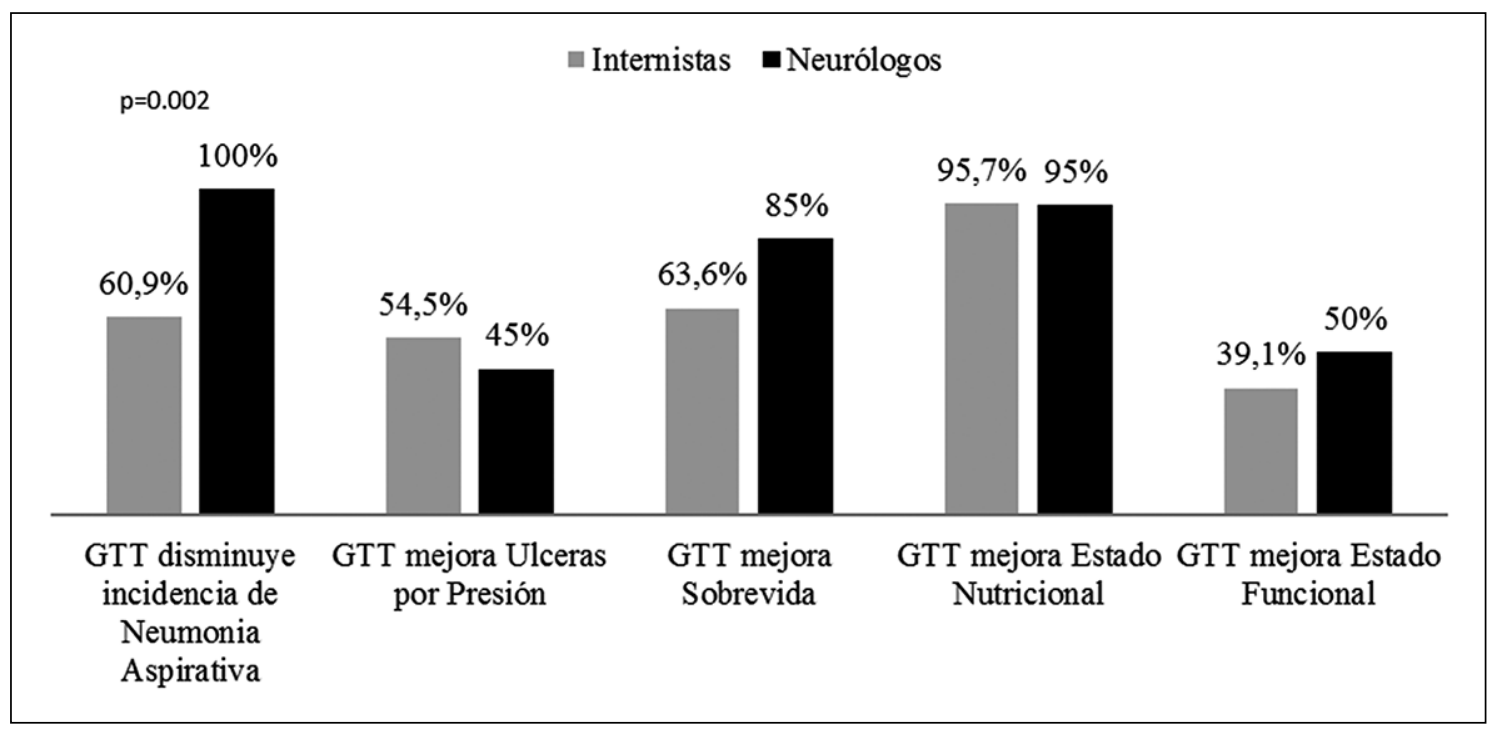

Figura 2. Resumen de opiniones sobre beneficios de gastrostomía en pacientes con demencia avanzada según especialidad.

Tabla 2. Frecuencia de respuestas sobre factores que consideran los médicos al indicar GTT en pacientes con demencia avanzada

\begin{tabular}{|lcc|}
\hline & Sí (\%) & No (\%) \\
\hline Voluntades anticipadas del paciente & 63,2 & 36,8 \\
\hline Preferencias de la familia & 94,9 & 5,1 \\
\hline Estado cognitivo del paciente & 94,4 & 5,6 \\
\hline Pronóstico del paciente & 94,9 & 5,1 \\
\hline Funcionamiento físico del paciente & 92,3 & 7,7 \\
Edad del paciente & 79,5 & 20,5 \\
\hline Costo de GTT & 53,8 & 46,2 \\
\hline
\end{tabular}

aspecto se aprecia una diferencia significativa por especialidad.

Entre los participantes, $61,9 \%$ opinó que los pacientes con demencia avanzada no sufren hambre ni sed al dejar de alimentarse por vía oral, $73,8 \%$ cree que son candidatos a institucionalizarse y $76,7 \%$ refiere que las creencias religiosas de los médicos no influyen en la decisión de indicar GTT. El 51,2\% opina que la GTT es parte del cuidado básico. Sólo se observaron diferencias significativas en este último aspecto entre neurólogos e internistas: los primeros opinan que la GTT es $(\mathrm{p}=0,021)$ y debiera ser parte del cuidado habitual $(\mathrm{p}=0,043)$ enpacientes con demencia avanzada.

En relación al rol del fonaudiólogo, 100\% de los internistas pide la evaluación por este profesional, mientras que $68 \%$ de los neurólogos lo hace $(\mathrm{p}=0,002)$. El 85\% de los médicos participantes, refirieron que el fonoaudiólogo les ha recomendado instalar GTT en pacientes con demencia avanzada y 95,3\% de los médicos señaló que las recomendaciones del fonoaudiólogo han influido en su decisión.

El 56,1\% de los médicos afirmó que el equipo de nutrición del lugar donde trabajan recomienda GTT. El 83,7\% consideran que las enfermeras o cuidadores tienen influencia en la decisión de indicar GTT a pacientes con demencia. El 74,4\% considera que la opinión de la enfermera o cuidadores acerca de la instalación de GTT influye en la opinión de la familia del paciente (Tabla 3).

\section{Discusión}

La población de adultos mayores continuará aumentando en Chile y, por lo tanto, aumentará también la incidencia de demencia. Si bien es cierto, que el soporte nutricional a través de una sonda de GTT percutánea puede ser beneficiosa en muchos pacientes, su uso en pacientes con 
Tabla 3. Influencias de creencias del médico, presión del personal de salud, y restricciones del sistema en la discusión de GTT

\begin{tabular}{|c|c|c|}
\hline & Sí (\%) & No (\%) \\
\hline \multicolumn{3}{|l|}{ Creencias personales } \\
\hline ¿Cree que el paciente sufre hambre y sed cuando deja de alimentarse por vía oral? & 61,9 & 38,1 \\
\hline ¿Cree que la GTT es parte del cuidado estándar? & 51,2 & 48,8 \\
\hline ¿Cree que los pacientes con demencia avanzada son candidatos a institucionalizarse? & 73,8 & 26,2 \\
\hline ¿Sus opciones religiosas influyen en la decisión de recomendar GTT? & 76,7 & 23,3 \\
\hline \multicolumn{3}{|l|}{ Presión del personal de salud } \\
\hline ¿Los fonoaudiólogos recomiendan GTT en demencia avanzada? & 85 & 15 \\
\hline ¿Las recomendaciones del fonoaudiólogo influyen en su decisión? & 95,3 & 4,7 \\
\hline ¿La opinión de las enfermeras influyen en su decisión? & 83,7 & 16,3 \\
\hline ¿Las enfermeras influyen en la familia sobre la instalación de GTT? & 74,4 & 25,6 \\
\hline ¿El Equipo de Nutrición recomienda GTT? & 56,1 & 43,9 \\
\hline \multicolumn{3}{|l|}{ Otros } \\
\hline ¿Las casas de reposo han solicitado instalación de GTT? & 11,6 & 88,4 \\
\hline ¿Las preocupaciones de la casa de reposo han influido en su decisión? & 54,8 & 45,2 \\
\hline ¿Ha sentido riesgo de mala práctica si no instala GTT? & 19 & 81 \\
\hline ¿Tiene tiempo para discutir los riesgos y beneficios de la GTT? & 90,2 & 9,8 \\
\hline
\end{tabular}

demencia avanzada es cuestionado y puede generar problemas éticos de proporcionalidad de tratamiento. Los pacientes con demencia avanzada frecuentemente desarrollan trastornos de deglución, dificultades en la alimentación o indiferencia ante la comida, llevando a una reducción de la ingesta, pérdida de peso y aumento del riesgo de neumonía aspirativa ${ }^{8}$. Esta situación es propia de etapas avanzadas de la enfermedad y la alimentación por sonda no ha demostrado compensar estos déficits ni prolongar la vida ${ }^{9}$. En relación al beneficio de esta vía de alimentación, a pesar de que la revisión sistemática de evidencia científica no apoya las posibles ventajas ${ }^{8}$ y que $44,2 \%$ de los encuestados refirió haber revisado literatura de demencia avanzada, 79,1\%de los médicos entrevistados considera que la GTT disminuye la incidencia de neumonía aspirativa, $50 \%$ opina que mejora las úlceras por presión, $73,8 \%$ que mejora la sobrevida, $95,3 \%$ que mejora el estado nutricional y $44,2 \%$ que mejora el estado funcional. $\mathrm{Al}$ comparar la muestra por subsistema de trabajo y por especialidad, se observó diferencias en relación a los criterios sobre la incidencia de neumonía aspirativa y de otros posibles beneficios relacionados a laGTT. Esto podría deberse a que existen otro tipo de pacientes que sí se benefician con la GTT (pacientes con accidente cerebrovasculares o con algunas enfermedades neurodegenerativas) lo cual genera malentendidos en los profesionales o en las familias relacionados a las expectativas de instalar esta vía de alimentación en estos pacientes. De hecho los riesgos pueden ser mayores que los potenciales beneficios al tratar de mejorar la nutrición mediante GTT $^{5,9}$ y existe diversidad de opiniones entre los médicos tratantes de este tipo de pacientes.

En el caso de pacientes con demencia avanzada, no existe consenso entre los encuestados en cuanto a que la GTT sea una indicación terapéutica o parte del cuidado básico o estándar. Otro problema se refiere a la sensación de hambre. El $38,1 \%$ de los médicos entrevistados considera que los pacientes sienten hambre y sed cuando dejan de alimentarse por vía oral, pero hay que tener en cuenta que la anorexia en el período terminal es un síntoma frecuente sin sensación de hambre. Por otro lado, se ha señalado que la deshidratación sistémica preterminal no sería desagradable para el paciente ${ }^{10}$. Probablemente el problema surge porque no sabemos cuándo los pacientes con demencia avanzada se encuentran ya próximos a la muerte.

Desde una mirada bioética los esfuerzos por prolongar la vida deben constituir un beneficio real para el paciente, y no instalarse sólo porque sean técnicamente posibles. Para ello es necesario pensar críticamente si es razonable y prudente 
prolongar la vida biológica cuando la vida biográfica ya no es posible. Algunos incluso podrían considerar la instalación de GTT en pacientes con demencia avanzada como una práctica maleficiente de obstinación terapéutica. De acuerdo a la literatura ${ }^{5,9}$, no existe evidencia clara sobre los beneficios de la GTT en la calidad de vida de pacientes con demencia avanzada. Más aún, el hecho de instalar GTT se asocia con mayor necesidad de contenciones físicas ya que los pacientes pueden intentar sacarse la sonda, disminuye el contacto con el resto de la familia o con los cuidadores, y se pierde una sensación básica como es sentir sabores. Hay que tener presente que la alimentación oral es una de las formas de mantener algún tipo de conexión con el medio y, generalmente, se puede entrenar a familiares y cuidadores para lograr una alimentación oral más segura.

Como último punto a analizar, parece relevante mencionar que $85 \%$ de los médicos entrevistados considera que los fonoaudiólogos recomiendan GTT en pacientes con demencia avanzada, por lo que también en esta profesión es necesaria una revisión del tema y un análisis crítico de cada situación en particular y tener presente los resultados de la instalación de GTT en estos pacientes $^{11}$. Existen evidencias que demuestran que los pacientes y sus familiares suelen aceptar lo que sugiere el personal sanitario, y no sólo el médico, por lo tanto, la responsabilidad de esta indicación recae en todo el equipo de salud ${ }^{10}$.

Finalmente, aunque el tamaño de la muestra de este estudio no permite obtener conclusiones generalizables, se observan criterios claros en las respuestas. Las decisiones sobre el uso de métodos alternativos de alimentación, sobre todo la instalación de GTT en pacientes con demencia avanzada, son complejas y algunas veces controvertidas. A pesar de que la literatura sugiere escaso o ningún beneficio asociado a la alimentación por GTT en pacientes en etapa avanzada de demencia, la mayoría de los médicos entrevistados cree que la instalación de GTT los podría beneficiar. Se propone que los profesionales involucrados en las decisiones y en la instalación de GTT en este tipo de pacientes se replanteen críticamente los criterios de su indicación y sus reales beneficios.

Agradecimientos: Dr. Juan Pablo Beca I., Socióloga M. Isabel Matute W.

\section{Referencias}

1. Banco Mundial. Esperanza de vida al nacer, total. En: http://datos.bancomundial.org/indicador/SP.DYN. LE00,IN. [Consultado el 28 de enero de 2014].

2. Gajardo J, Monsalves M. Demencia, un tema urgente para Chile. Rev Chil Salud Pública 2013; 17 (1): 22-5.

3. Barroso J. Disfagia Orofaríngea y Broncoaspiración, Rev Esp Geriatr Gerontol 2009; 44 (52): 22-8.

4. Mendiratta P, Tilford J, Prodhan P, Curseen K, Azhar G, Eis J. Trends in Percutaneous Endoscopic Gastrostomy Placement in the Elderly From 1993 to 2003. Am J of Alzheimers Dis Other Demen 2001; 27 (8): 609-13.

5. Cervo F, Bryan L, Farber Sh. To PEG or not to PEG. A review of evidence for placing tubes in advanced dementia and the decision making process. Geriatrics 2006; 61 (6): 30-5.

6. Sharp H, Shega J. Feeding Tube Placemente in Patients With Advanced Dementia: The Beliefs and Practice Patterns of Speech-Language Pathologistis. Am J Speech Lang Pathol 2009; 18 (3): 222-30.

7. Shega J, Hougham G, Stocking C, Cox-Hayley D, Sachs G. Barriers to Limiting the Practice of Feeding Tube Placement in Advanced Dementia. J Palliat Med 2003; 6 (6): 885-93.

8. Sherman F. Nutrition in advanced dementia. Tube feeding or hand-feeding until death? Geriatrics 2003; 58 (11): 10-2.

9. Sampson E, Candy B, Jones L. Enteral tube feeding for older people with advanced dementia. Cochrane Database of Systematic Reviews 2009, Issue 2. Art. No.: CD007209. DOI: 10.1002/14651858.CD007209.pub2.

10. Martínez J, Tapiera A, Blasco M. Decisiones ético-clínicas sobre la alimentación e hidratación artificial mediante sonda en la enfermedad terminal. Nutr Hosp 2002; 17 (6): 279-83.

11. Galicia-Castillo M. The PEG dilema. Feeding tubes are not the answer in advanced dementia. Geriatrics 2006; 61 (6): 12-3. 